\title{
Field-induced orientational percolation to a ferroelectric phase in relaxor $\mathrm{Pb}\left(\operatorname{In}_{1 / 2} \mathrm{Nb}_{1 / 2}\right)_{1-x} \operatorname{Ti}_{x} \mathrm{O}_{3}$
}

\author{
C.-S. Tu, ${ }^{1, *}$ R. R. Chien, ${ }^{2}$ C.-M. Hung, ${ }^{1}$ V. Hugo Schmidt, ${ }^{2}$ F.-T. Wang, ${ }^{1}$ and C.-T. Tseng ${ }^{1}$ \\ ${ }^{1}$ Graduate Institute of Applied Science and Engineering, Fu Jen Catholic University, Taipei 242, Taiwan, Republic of China \\ ${ }^{2}$ Department of Physics, Montana State University, Bozeman, Montana 59717, USA
}

(Received 2 March 2007; published 5 June 2007)

\begin{abstract}
This work presents direct observations of orientational percolation and depolarization in a (001)-cut relaxor $\mathrm{Pb}\left(\mathrm{In}_{1 / 2} \mathrm{Nb}_{1 / 2}\right)_{0.70} \mathrm{Ti}_{0.30} \mathrm{O}_{3}$ (PINT30\%) crystal by means of dielectric permittivity, depolarization current, domain structure, and hysteresis loop. Electric $(E)$-field poling induced a $10-300 \mu \mathrm{m}$ size orientational percolation transition in the matrix. The percolation breaks down at depolarization temperature $T_{d} \cong 395 \mathrm{~K}$, which is $\sim 20-40 \mathrm{~K}$ higher than in rhombohedral $\mathrm{Pb}\left(\mathrm{Mg}_{1 / 3} \mathrm{Nb}_{2 / 3}\right)_{1-x} \mathrm{Ti}_{x} \mathrm{O}_{3}$. Near $420 \mathrm{~K}$, the remaining polarization disappears, and the full relaxor-type dielectric dispersion due to polar nanoclusters reappears, and remains evident up to the Burns temperature $T_{B} \cong 520 \mathrm{~K}$. Under $E=40 \mathrm{kV} / \mathrm{cm}$ at room temperature, small regions of [001] tetragonal domains appeared, but most of the crystal exhibited field-induced percolation rhombohedral and monoclinic microdomains embedded randomly in the matrix. This work suggests that this relaxor ferroelectric consists of two components (spherical glassy matrix and polar nanoclusters) as proposed for $\mathrm{Pb}\left(\mathrm{Mg}_{1 / 3} \mathrm{Nb}_{2 / 3}\right) \mathrm{O}_{3}$ by Blinc et al. [Phys. Rev. Lett. 91, 247601 (2003)].
\end{abstract}

DOI: $10.1103 /$ PhysRevB.75.212101

PACS number(s): 77.80.Bh, 77.80.Dj, 77.84.-s

The most important nature of relaxor ferroelectrics probably is the existence of polar nanoclusters [or polar nanoregions (PNRs)] which are believed to be responsible for many physical properties, such as dielectric relaxation behavior, high piezoelectricity, and phase instability. Field-cooled (FC) and zero-field-cooled (ZFC) ${ }^{207} \mathrm{~Pb}$ nuclear-magneticresonance (NMR) spectra of the prototype relaxor $\mathrm{Pb}\left(\mathrm{Mg}_{1 / 3} \mathrm{Nb}_{2 / 3}\right) \mathrm{O}_{3}$ (PMN) crystal show the existence of two components-spherical glassy matrix and ferroelectric nanoclusters. ${ }^{1,2}$ About $50 \%$ of the $\mathrm{Pb}$ nuclei reside in the spherical glassy matrix which does not respond to the external field, and $50 \%$ in the ferroelectric polar nanoclusters which can respond to $E$ field (greater than threshold field $E_{t}$ ). Below $T_{C} \cong 210 \mathrm{~K}$, a sudden intensity increase in the anisotropic NMR line corresponding to polar nanoclusters was evidenced in the ZFC spectra, but the magnitude is two times smaller than in the FC spectra $\left(E>E_{t} \cong 1.8 \mathrm{kV} / \mathrm{cm}\right) .^{2}$ An extra dielectric peak was observed near $210 \mathrm{~K}$ in the fieldheated and/or field-cooled spectra, implying a first-order ferroelectric transition. ${ }^{3}$ Neutron analysis of unpoled PMN powder shows that the volume fraction of PNRs and their correlation lengths increase drastically below $200 \mathrm{~K} .{ }^{4}$ These evidences indicate that PMN is incipient ferroelectric. Recent first-principles molecular-dynamics simulations for $\mathrm{Pb}\left(\mathrm{Sc}_{1 / 2} \mathrm{Nb}_{1 / 2}\right) \mathrm{O}_{3}$ and $\mathrm{PMN}$ show formations of nanoclusters in the quenched short-range-ordered regions below the Burns temperature..$^{5}$ By dielectric and domain studies, incipient ferroelectric natures were also found in $\mathrm{Pb}\left(\mathrm{Mg}_{1 / 3} \mathrm{Nb}_{2 / 3}\right)_{1-x} \mathrm{Ti}_{x} \mathrm{O}_{3}$ (PMNTx) crystals $(x=24 \%-38 \%)$, in which a "hidden" transition (which was seen in unpoled samples but not as clearly as in the poled sample) was enhanced by an $E$ field. ${ }^{6}$ This hidden transition is associated with the appearance of monoclinic $(M)$ microdomains.

High-strain ferroelectric crystals PMNT and $\mathrm{Pb}\left(\mathrm{Zn}_{1 / 3} \mathrm{Nb}_{2 / 3}\right)_{1-x} \mathrm{Ti}_{x} \mathrm{O}_{3}$ have demonstrated their values in piezoelectric devices, ${ }^{7}$ and their physical properties are sensitive to Ti content, $E$-field poling, crystallographic orientation, annealing, and other factors. ${ }^{6,8-10}$ However, thermal instability (variation with temperature) caused by overheating and $E$-field-induced fracture remain problems in use of these materials. It has been a goal to find high $T_{C}$ or $T_{d}$ (Curie or depolarization temperature) crystals. $T_{d}$ represents the temperature in a prior-poled zero-field-heated experiment at which the local polarization exhibits a large and sudden reduction by thermal energy. Among high-strain materials, $\mathrm{Pb}\left(\mathrm{In}_{1 / 2} \mathrm{Nb}_{1 / 2}\right)_{1-x} \mathrm{Ti}_{x} \mathrm{O}_{3}$ (PINTx) has drawn attention because of its high- $T_{C}$ feature. ${ }^{11-17}$ From dielectric results, temperature- and $E$-field-dependent phase diagrams were proposed for PINT crystals and ceramics. ${ }^{11,12}$ The (001)-cut PINT34\%-35\% crystals have piezoelectric constant $d_{33}$ $\cong 2000 \mathrm{pC} / \mathrm{N}$ and electromechanical coupling factor $k_{33}$ $\cong 94 \%$ with $T_{C} \cong 270{ }^{\circ} \mathrm{C} .{ }^{13,14}$ A diffuse field-induced rhombohedral $(R) \rightarrow$ tetragonal $(T)$ transition was seen in a (001)cut PINT28\% crystal. $^{15}$ The unpoled (001)-cut PINT30\% crystal exhibits a broad dielectric dispersion which can be described by the Vogel-Fulcher equation. ${ }^{16}$

In this study, the single crystal PINT30\% (starting composition) was grown using a modified Bridgman method with an allomeric PMNT29\% seed crystal to restrain the pyrochlore phase. ${ }^{17}$ A Wayne-Kerr analyzer PMA3260A was used to obtain the real part $\varepsilon$ ' of dielectric permittivity. The sample was cut perpendicular to a $\langle 001\rangle$ direction and was coated with gold electrodes. Three processes were used in the dielectric studies. The first two are called "zero-fieldheated" (ZFH) and "zero-field-cooled" (ZFC), in which the data were taken upon heating and cooling without any poling. In "zero-field-heated after prior poled" (PP-ZFH), the sample was poled at room temperature with a dc $E$ field of $5 \mathrm{kV} / \mathrm{cm}$ along [001], then cooled to $150-200 \mathrm{~K}$ without an $E$ field before ZFH was performed. An irregular piezoelectric resonance was observed for $f>100 \mathrm{kHz}$ in the PP-ZFH dielectric spectra. PP-ZFH depolarization current was measured by using a Keithley 6517A electrometer. Hysteresis loops were taken by using a Sawyer-Tower circuit at $f$ $=46 \mathrm{~Hz}$. A Janis CCS-450 cold head was used with a LakeShore 340 temperature controller. 


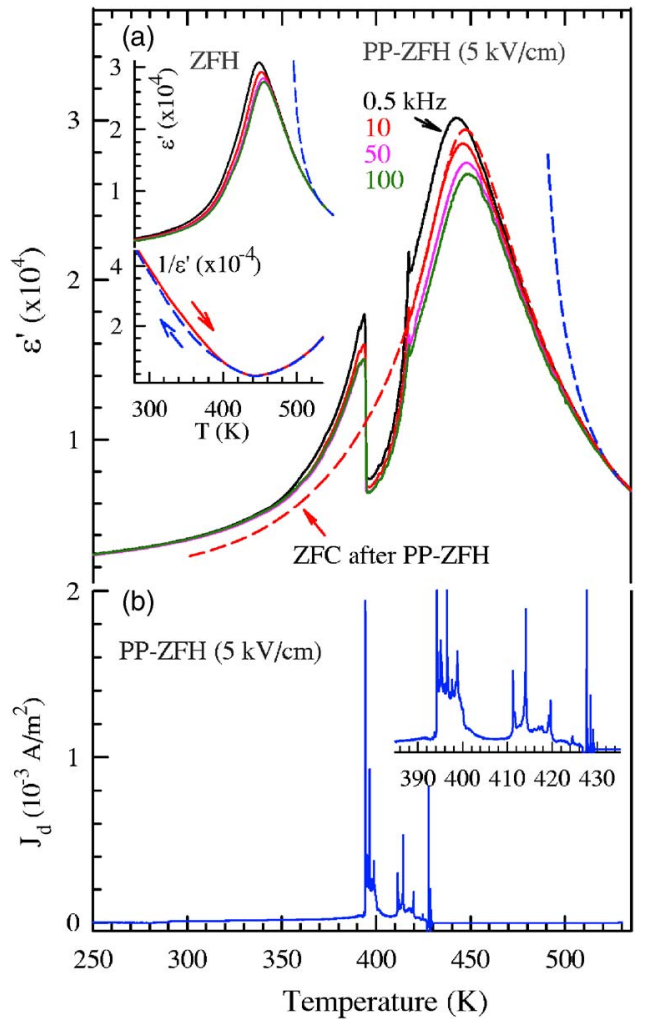

FIG. 1. (Color online) (a) PP-ZFH dielectric permittivity and (b) PP-ZFH depolarization current density $J_{d}$. The red dashed curve indicates the ZFC result after PP-ZFH. The insets of (a) are $\varepsilon^{\prime}$ (ZFH) and $1 / \varepsilon^{\prime}(\mathrm{ZFC} / \mathrm{ZFH})$ taken at $f=10 \mathrm{kHz}$. The inset of (b) is an enlargement of depolarization regions.

Domain structures were observed by using a Nikon E600POL polarizing microscope with a crossed polarizer/ analyzer (P/A) pair. Transparent conductive films of indium tin oxide were deposited on the (001) basal surfaces. The crystal thickness is about $70 \mu \mathrm{m}$. Angles of the P/A pair measured in this work are with regard to the [110] direction. The experimental setup and details for using optical extinction to determine domain phases can be found in Ref. 9 .

Figure 1 shows (a) dielectric permittivity and (b) depolarization current density obtained from PP-ZFH and ZFH processes. Diffuse frequency-dependent maxima arise in the region of $430-460 \mathrm{~K}$ and shift toward higher temperatures with frequency. In the PP-ZFH, an extra dielectric anomaly (that was not seen in the ZFH) occurs at $T_{d} \cong 395 \mathrm{~K}$, below which the frequency dispersion almost disappears, but is still visible. The threshold $E$ field to induce this extra anomaly is about $E_{t}=1 \mathrm{kV} / \mathrm{cm}$ which is smaller than the coercive field $E_{C} \cong 4.5 \mathrm{kV} / \mathrm{cm}$ at room temperature. As seen in the inset of Fig. 1(a), a clear thermal hysteresis begins to develop near $395 \mathrm{~K}$, implying a first-order ferroelectric transition. In Fig. 1(b), the current density exhibits strong multiple Barkhausen-noise-like jumps in the region of 390-400 K. The depolarization current density $\left(J_{d}=-d \vec{P} / d t\right)$ is mainly associated with bound surface $\left(\sigma_{b}=\vec{P} \cdot \hat{n}\right)$ and bound volume $\left(\rho_{b}=-\nabla \cdot P\right)$ charge densities which are functions of local polarization $P$. This dramatic current response confirms a dy- namic reorganization of polarization directions and correlation lengths of local domains. Multiple jumps of depolarization current can be interpreted as discrete changes in the size or rotation of ferroelectric domains due to breakup of $10-300 \mu \mathrm{m}$ size microdomains into variously orieted smaller microdomains or nanodomains. The multiple-jump response of the depolarization current was also seen in (111)cut PMN and PMNT12\% crystals after poling. ${ }^{18}$

As the temperature increases in PP-ZFH above $T_{d}$ $\cong 395 \mathrm{~K}$, the dielectric dispersion remains weak as $\epsilon^{\prime}$ rises more and more rapidly. The full frequency spread seen in ZFH suddenly reappears in PP-ZFH near $420 \mathrm{~K}$. This sudden transition behavior correlates with that seen in the inset of Fig. 1(b), where the current density exhibits a complicated multiple-jump response in the region of $410-430 \mathrm{~K}$. These phenomena indicate another polarization reorganization, but its magnitude is smaller than the response in the region of 390-400 K. $\epsilon^{\prime}(T)$ was found to follow the Curie-Weiss equation, $\epsilon^{\prime}(T)=C /\left(T-T_{0}\right)$, in the cubic phase above $510 \mathrm{~K}$. The blue dashed lines are fittings with $C=3.9 \times 10^{5}$ and $T_{0}$ $=472 \mathrm{~K}$ for $\mathrm{ZFH}$ and $C=3.9 \times 10^{5}$ and $T_{0}=477 \mathrm{~K}$ for PP$\mathrm{ZFH}$. Noticeable deviations from the Curie-Weiss law start from $\sim 510$ for ZFH and $\sim 520 \mathrm{~K}$ for PP-ZFH. We consider 510 and $520 \mathrm{~K}$ to be the Burns temperatures $\left(T_{B}\right)$, below which polar nanoclusters begin to develop. Their weaker dielectric response causes deviations from the Curie-Weiss law and their dynamics is responsible for the diffuse dielectric dispersion. ${ }^{19}$ As shown by the red dashed curve in Fig. 1(a), the ZFC dielectric permittivity measured after the PP-ZFH does not show the dielectric anomaly in the region of $390-430 \mathrm{~K}$. This indicates that the poling effect (or the polarized state) can be erased by thermal annealing.

Figure 2 shows temperature-dependent (a) hysteresis loops and (b) remanent polarization $\left(P_{r}\right)$ and coercive field $\left(E_{C}\right)$ versus temperature. The spontaneous polarization $\left(P_{S}\right)$, $P_{r}$, and $E_{C}$ are $\sim 21 \mu \mathrm{C} / \mathrm{cm}^{2}, \sim 20 \mu \mathrm{C} / \mathrm{cm}^{2}$, and $\sim 4.5 \mathrm{kV} / \mathrm{cm}$ at room temperature, respectively. As shown in Fig. 2(b), both $P_{r}$ and $E_{C}$ exhibit a clear dip near $380 \mathrm{~K}$, implying a significant polarization reorganization of local domains. $380 \mathrm{~K}$ is fairly consistent with the temperature where the extra dielectric anomaly and the strong current response appear in the PP-ZFH.

Before an $E$-field poling as shown in Fig. 3(a), various interference-color domains (whose sizes are less than $10 \mu \mathrm{m})$ distribute randomly in the matrix with optical extinction at $\mathrm{P} / \mathrm{A}: 0^{\circ}$, indicating rhombohedral $(R)$ and monoclinic $\left(M_{A^{-}}\right.$or $\left.M_{B^{-}}\right)$symmetries according to the extinction principles of polarizing microscopy. ${ }^{9}$ This coexistence of $R$ and $M$ phases is confirmed by the $\langle 002\rangle$ x-ray diffraction in Fig. 4 which shows a sharp peak ( $R$ phase) and a broad shoulder ( $M$ phase). A similar x-ray spectrum of $R$ and $M$ phase coexistence was observed in PMNT31\% ceramic. ${ }^{8}$ As the temperature increases as shown in Fig. 3(b), the interference color of part of the matrix exhibits an obvious change in the region of 385-395 K without changing extinction angle. Note that a thermal hysteresis begins to develop near $395 \mathrm{~K}$ in ZFH [Fig. 1(a)].

ZFH domain structures after poling at $E=5 \mathrm{kV} / \mathrm{cm}$ along [001] are illustrated in Figs. 3(c)-3(g) from 298 to $453 \mathrm{~K}$. 

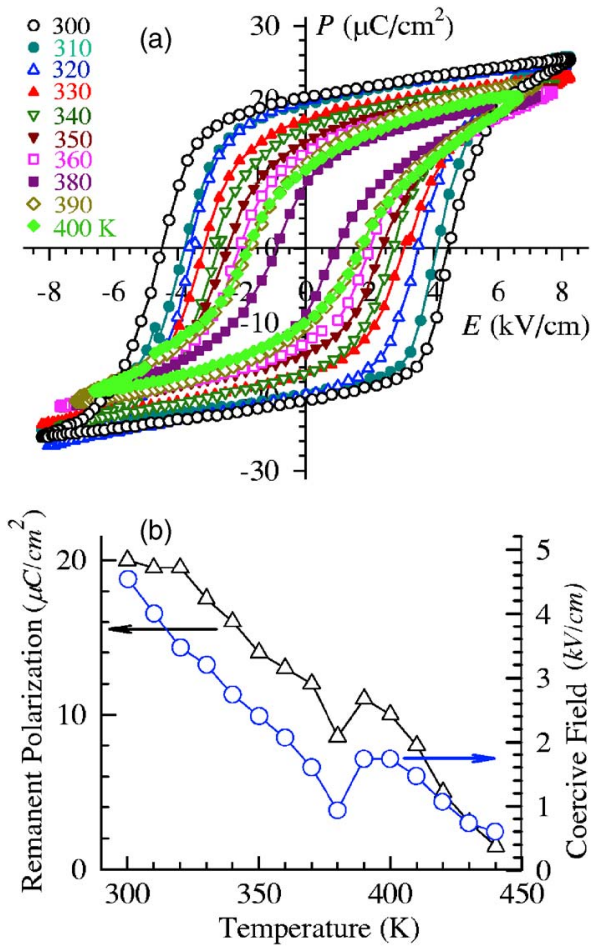

FIG. 2. (Color online) Temperature-dependent (a) hysteresis loop and (b) remanent polarization and coercive field upon heating.

After poling at $298 \mathrm{~K}$, percolating microdomains of approximately $10-300 \mu \mathrm{m}$ sizes were induced with the same extinction angle of $\mathrm{P} / \mathrm{A}: 0^{\circ}$, especially in the region as encircled in Fig. 3(c). These $10-300 \mu \mathrm{m}$ size microdomains distribute randomly in the matrix, implying that only part of the matrix can respond to an external $E$ field. The field-induced micrometric percolations were caused by rotating much smaller microdomain or nanocluster polarizations into alignment so that the boundaries disappear. The $\langle 002\rangle \mathrm{x}$-ray diffraction (Fig. 4) shows that the ratios of the intensities of the shoulder and the sharp peak are almost the same before and after poling at $E=5 \mathrm{kV} / \mathrm{cm}$, which means that amounts of the $R$ and $M$ domains do not change by $E$ field poling. As the temperature increases, the birefringence of the 10-300 $\mu \mathrm{m}$ size percolating microdomains becomes small radically near $391 \mathrm{~K}$, as shown by the gray interference color, and has the same extinction at P/A:0 $0^{\circ}$ as given in Figs. 3(d) and 3(e). These gray regions most likely are associated with the appearance of very small microdomains and/or polar nanoclusters expand quickly above $391 \mathrm{~K}$. Note that the optimal resolution of the polarizing microscope is about $1 \mu \mathrm{m}$ due to the optical diffraction limit. As seen in Figs. 3(f) and 3(g), the domain matrix becomes darker with increasing temperature. No obvious domain anomaly was observed around $410-430 \mathrm{~K}$ which corresponds to the secondary depolarization anomaly and reappearance of strong dielectric dispersion (Fig. 1).

The above results reveal that a breakdown of 10-300 $\mu \mathrm{m}$ size percolating microdomains occurs at $T_{d} \cong 395 \mathrm{~K}$, where polar nanoclusters begin to establish and thus reduce the averaged optical birefringence. The rapid spread of grayinterference-color regions in $395-420 \mathrm{~K}$ indicates a growing
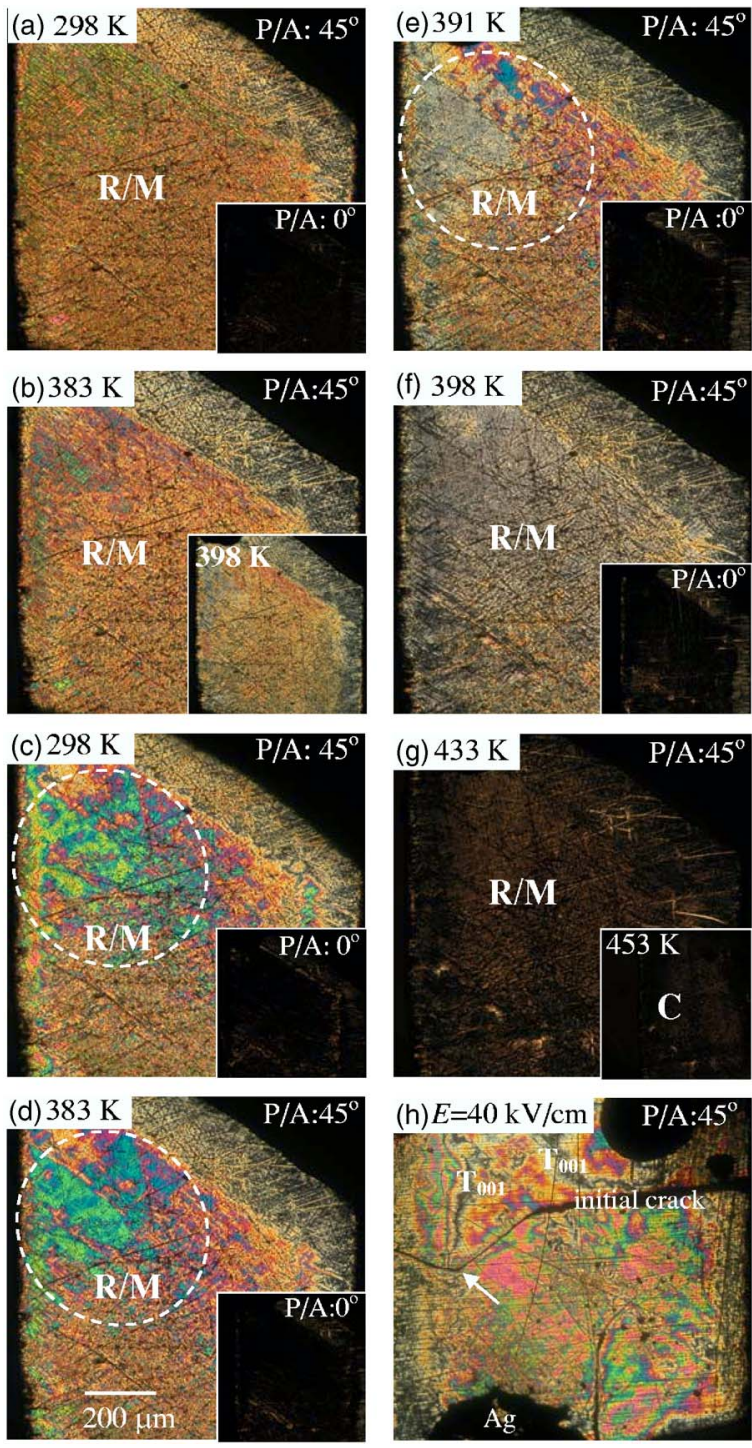

FIG. 3. (Color online) Domain structures [(a) and (b)] from 298 to $398 \mathrm{~K}$ before poling, [(c)-(g)] from 298 to $453 \mathrm{~K}$ after poling $(E=5 \mathrm{kV} / \mathrm{cm})$, and (h) under $E=40 \mathrm{kV} / \mathrm{cm}$ along [001] at room temperature.

integration of polar nanoclusters, whose dynamics causes the recovery of dielectric relaxation. The domain matrix displays a total optical extinction near $453 \mathrm{~K}$ and enters the cubic $(C)$ phase, as seen in the inset of Fig. $3(\mathrm{~g})$. The crystal is in a micrometric cubic state for $T \geqslant 453 \mathrm{~K}$ with existence of polar nanoclusters which vanish at $T_{B} \cong 520 \mathrm{~K}$.

Figure 3(h) shows the $E$-field-induced domain structure under $E=40 \mathrm{kV} / \mathrm{cm}$ along [001] at room temperature. Only a small portion of the domain matrix became the [001] tetragonal phase as indicated by $T_{001}$. Most of the crystal exhibits irregular-shaped field-induced percolation microdomains distributed randomly in the matrix. Most areas of the domain matrix have an extinction angle of $\mathrm{P} / \mathrm{A}: 0^{\circ}$, indicating mostly $R$ and some $M$ domains. An internal crack (as indicated by the arrow) starts to expand notably along [100] and [010] directions from the initial crack (caused by polishing) at $E$ $\cong 24 \mathrm{kV} / \mathrm{cm}$. Similar $E$-field-induced domain structures 


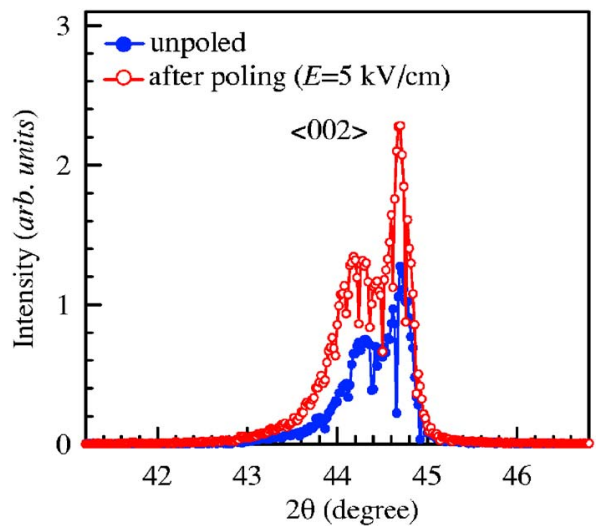

FIG. 4. (Color online) $\langle 002\rangle \mathrm{x}$-ray-diffraction spectra before and after poling $(E=5 \mathrm{kV} / \mathrm{cm})$.

were observed in the (001)-cut PMNT24\% crystal, in which percolating spotlike microdomains embed randomly in the matrix and the crystal cannot entirely reach the $T_{001}$ phase under $E=44 \mathrm{kV} / \mathrm{cm}^{9}$

In conclusion, this Brief Report shows direct evidences of orientational percolation into $10-300 \mu \mathrm{m}$ size microdomains induced by an $E$ field. With increasing temperature, they break down into polar nanoclusters whose dynamics reestablishes the dielectric relaxation. This breakdown (thermal depolarization) occurs in two narrow temperature regions. It starts at $T_{d} \cong 395 \mathrm{~K}$ due to the micrometric percolation breakdown into polar nanoclusters. The second depolarization range is near $410-430 \mathrm{~K}$. The reason for two steps at 395 and $410 \mathrm{~K}$ and the behavior between these tem- peratures are not fully understood. This rapid growth of polar nanoclusters in the region of 390-430 K ends with reestablishment of the full-scale diffuse dielectric relaxation above $420 \mathrm{~K}$. The crystal is in a micrometric cubic state near $450 \mathrm{~K}$ with existence of polar nanoclusters which vanish above $T_{B}$ $\cong 520 \mathrm{~K}$. The depolarization temperature $T_{d} \cong 395 \mathrm{~K}$ of (001) PINT30\% crystal is about $20^{\circ}-40^{\circ}$ higher than rhombohedral PMNT24\% and 30\% crystals, ${ }^{20}$ which is promising for applications. This work suggests that moderate $E$-field poling does not create a new phase, but it can induce microscopic or macroscopic percolations in the matrix. This study also presents some microscopic confirmation of ${ }^{207} \mathrm{~Pb} \mathrm{NMR}$ result, which proposes two components in the prototype PMN crystal, i.e., spherical glassy matrix and polar nanoclusters. ${ }^{2}$ The polar (ferroelectric) nanoclusters are embedded in the glassy matrix and can be orientationally percolated into micrometric clusters by an external $E$-field $\left(\geqslant E_{t} \cong 1.0 \mathrm{kV} / \mathrm{cm}\right.$ ). The glassy matrix (probably the material in stronger local fields) does not respond to the $E$ field, but it is responsible for some of the wide-temperature-range dielectric relaxation which can even be seen below $T_{d}$ in the PP-ZFH. Based on domain results, the PINT30\% crystal shows an incipient ferroelectric nature, in which a "hidden" transition was seen in the unpoled sample (but not as obvious as in the poled sample). Incipient ferroelectric nature is a common phenomenon in relaxor ferroelectrics.

The authors would like to thank H. Luo (Shanghai Institute of Ceramics) for crystals. This work was supported by National Science Council of Taiwan Grant No. 95-2112-M030-001.
*Author to whom correspondence should be addressed. Email address: 039611@mail.fju.edu.tw

${ }^{1}$ R. Blinc, V. V. Laguta, and B. Zalar, Phys. Rev. Lett. 91, 247601 (2003).

${ }^{2}$ R. Blinc, V. V. Laguta, B. Zalar, and J. Banys, J. Mater. Sci. 41, 27 (2006).

${ }^{3}$ R. Sommer, N. K. Yushin, and J. J. van der Klink, Phys. Rev. B 48, 13230 (1993).

${ }^{4}$ I. K. Jeong, T. W. Darling, J. K. Lee, T. Proffen, R. H. Heffner, J. S. Park, K. S. Hong, W. Dmowski, and T. Egami, Phys. Rev. Lett. 94, 147602 (2005).

${ }^{5}$ S. Tinte, B. P. Burton, E. Cockayne, and U. V. Waghmare, Phys. Rev. Lett. 97, 137601 (2006).

${ }^{6}$ C.-S. Tu, R. R. Chien, F.-T. Wang, V. H. Schmidt, and P. Han, Phys. Rev. B 70, 220103(R) (2004).

${ }^{7}$ Y. Yamashita, Y. Hosono, K. Harada, and N. Yasuda, IEEE Trans. Ultrason. Ferroelectr. Freq. Control 49, 184 (2002).

${ }^{8}$ B. Noheda, D. E. Cox, G. Shirane, J. Gao, and Z.-G. Ye, Phys. Rev. B 66, 054104 (2002).

${ }^{9}$ R. R. Chien, V. H. Schmidt, C.-S. Tu, L.-W. Hung, and H. Luo, Phys. Rev. B 69, 172101 (2004).

${ }^{10}$ A. A. Bokov and Z.-G. Ye, J. Mater. Sci. 41, 31 (2006).
${ }^{11}$ N. Yasuda, H. Ohwa, K. Ito, M. Iwata, and Y. Ishibashi, Ferroelectrics 230, 115 (1999).

${ }^{12}$ E. F. Alberta and A. S. Bhalla, J. Korean Phys. Soc. 32, S1265 (1998).

${ }^{13}$ Y. Guo, H. Luo, T. He, X. Pan, and Z. Yin, Mater. Res. Bull. 38, 857 (2003).

${ }^{14}$ Z. Duan, G. Xu, X. Wang, D. Yang, X. Pan, and P. Wang, Solid State Commun. 134, 559 (2005).

${ }^{15}$ N. Yasuda, M. Sakaguchi, Y. Itoh, H. Ohwa, Y. Yamashita, M. Iwata, and Y. Ishibashi, Jpn. J. Appl. Phys., Part 1 42, 6205 (2003).

${ }^{16}$ C.-S. Tu, C.-M. Hung, F.-T. Wang, R. R. Chien, and S. W. Yang, Solid State Commun. 138, 190 (2006).

${ }^{17}$ Y. Guo, H. Luo, T. He, X. Pan, and Z. Yin, Solid State Commun. 123, 417 (2002).

${ }^{18}$ E. V. Colla and M. B. Weissman, Phys. Rev. B 72, 104106 (2005).

${ }^{19}$ D. Viehland, S. J. Jang, L. E. Cross, and M. Wuttig, Phys. Rev. B 46, 8003 (1992).

${ }^{20}$ Z. Feng, X. Zhao, and H. Luo, J. Appl. Phys. 100, 024104 (2006). 